\title{
Simple model for calculating the ratio of the carrier capture and escape times in quantum-well lasers
}

\author{
B. Romero, ${ }^{\text {a) }}$ J. Arias, I. Esquivias, and M. Cada ${ }^{\text {b) }}$ \\ Departamento de Tecnología Fotónica, E.T.S.I. Telecommunicación, Universidad Politècnica de Madrid, \\ Ciudad Universitaria, E-28040, Madrid, Spain
}

(Received 30 August 1999; accepted for publication 24 January 2000)

\begin{abstract}
We have developed a simple model for the carrier capture and escape processes in quantum-well (QW) lasers, which yields an analytical expression for the ratio of the carrier capture and escape times. It predicts a decrease in the escape time with injected carrier density due to the state filling effect. It also shows an exponential dependence of the escape time on the effective barrier height and on the inverse of the temperature. A comparison between experimental and calculated values for InGaAs/GaAs QW lasers is presented showing a good agreement. (C) 2000 American Institute of Physics. [S0003-6951(00)02412-8]
\end{abstract}

The carrier capture and escape times, $\tau_{\text {cap }}, \tau_{\text {esc }}$, are very important parameters that determine the static as well as the dynamic performance of quantum-well (QW) lasers. In dynamic operating conditions the capture and escape times affect both the resonant frequency and the damping rate of the modulation response, and thus determine the lasers' modulation bandwidth. ${ }^{1,2}$ Nowadays, most of the dynamic and static laser models include capture/escape effects ${ }^{3}$ without a clear knowledge of the values of the model parameters. It is, therefore, crucial to relate these values to the structural parameters of QW lasers and to the operating conditions. Experimentally, it has been observed that the escape time strongly depends on the depth of the $\mathrm{QW}$, on the level of the $p$ doping in the active region, as well as on the temperature and the bias current. $^{4}$

Different models have been proposed to calculate the capture and escape times in QW structures. In 1988, the thermionic emission model was developed to calculate the remission time from a QW (Ref. 5) under reverse-bias conditions using the Maxwell-Boltzmann statistics. Years later, the longitudinal optical (LO) phonon interaction theory was introduced by several groups ${ }^{6,7}$ to explain the mechanism by which a two-dimensional (confined) carrier interacts with a three-dimensional (unconfined) carrier. More sophisticated models, including different scattering mechanisms to account for the capture process, have also been proposed. ${ }^{8}$ However, those models rely on physical parameters that are not well known, and require complicated numerical calculations in order to obtain the time constants that are needed in the standard rate equations; therefore, they are not practical either for the simulation of phenomena which require large computational efforts (spectral and/or spatial effects), or for the design and optimization of new structures.

In this work, we propose a simple model for the interaction between confined and unconfined carriers which, under certain approximations, yields an analytical expression for the ratio of the capture and escape times. For the operating

\footnotetext{
${ }^{a)}$ Electronic mail: beatriz@tfo.upm.es

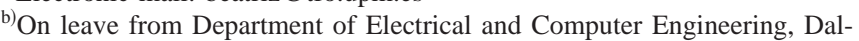
housie University, Halifax, N.S., B3J2X4, Canada.
}

conditions of most actual laser structures, our model predicts well the qualitative behavior obtained from more complex models that has also been observed experimentally: the capture time is constant with the injection level for a given structure while the escape time decreases with the injection level and shows an exponential dependence on the effective barrier height and on the inverse of the temperature. Finally, we provide a comparison between theoretical calculations and experimental results for the escape time in QW lasers.

The basis of the proposed model is to assume that transitions of carriers between unconfined and confined states are elastic, neglecting thus the energy difference between the initial and final states. Even though this assumption is not strictly valid, the model explains the main experimentally observed structural and operating-condition dependencies and it has the advantage of simplifying the calculations of $\tau_{\text {cap }}$ and $\tau_{\text {esc }}$. The analysis we present hereafter applies to electrons in the conduction band, although similar analysis can be carried out for holes in the valence band. Figure 1 shows the current flows coming into and out of the well; $I_{\text {cap }}$ is the capture current and $I_{\text {esc }}$ is the escape current. $I_{\text {cap }}\left(I_{\text {esc }}\right)$ is evaluated from the integral of the transition rate at each energy higher than the barrier band edge, considering that the transition rate is proportional to the number of filled unconfined (confined) states and to the number of empty confined (unconfined) states. The former can be expressed as

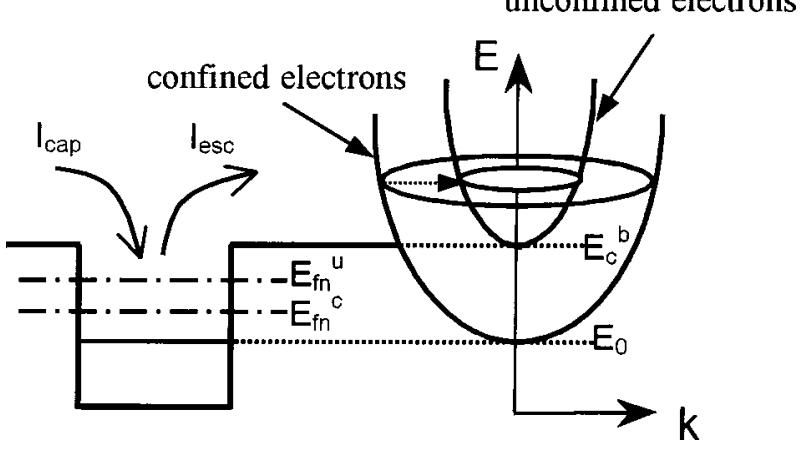

FIG. 1. Capture and escape currents in a QW laser structure, together with the conduction-band diagram. The dashed arrow represents an elastic interaction. 
TABLE I. Fabrication parameters, experimental data, ${ }^{a}$ and calculated values of $\tau_{\text {esc }}$ and $\tau_{\text {cap }} / \tau_{\text {esc }}$ for studied laser structures at a carrier density of $10^{18} \mathrm{~cm}^{-3} \cdot x_{\text {In }}$ and $N_{A}$ denote the In mole fraction and the nominal doping density in the QWs, respectively.

\begin{tabular}{cccccccc}
\hline \hline Sample & $x_{\text {In }}$ & $\begin{array}{c}N_{A} \\
\left(10^{18} \mathrm{~cm}^{-3}\right)\end{array}$ & $\begin{array}{c}\text { No. } \\
\text { QWs }\end{array}$ & $\begin{array}{c}\tau_{\text {esc }}(\exp ) \\
(\mathrm{ns})\end{array}$ & $\begin{array}{c}\tau_{\text {cap }} / \tau_{\text {esc }} \\
(\exp )\end{array}$ & $\begin{array}{c}\tau_{\text {esc }}(\text { calc) } \\
(\mathrm{ns})\end{array}$ & $\begin{array}{c}\tau_{\text {cap }} / \tau_{\text {esc }} \\
(\text { calc) }\end{array}$ \\
\hline A & 0.35 & $\cdots$ & 4 & $\sim 0.6$ & $<0.01$ & 0.8 & $7 \times 10^{-4}$ \\
B & 0.35 & 5 & 4 & $\sim 3$ & $<0.002$ & 3 & $2 \times 10^{-4}$ \\
C & 0.35 & 20 & 4 & $>2$ & $<0.002$ & 4 & $1 \times 10^{-4}$ \\
D & 0.25 & $\cdots$ & 1 & $<0.1$ & $\sim 0.8$ & 0.006 & 0.4 \\
E & 0.25 & $\ldots$ & 2 & $<0.1$ & $\sim 0.4$ & 0.006 & 0.2 \\
F & 0.25 & $\cdots$ & 3 & $<0.1$ & $\sim 0.2$ & 0.006 & 0.1 \\
\hline \hline
\end{tabular}

${ }^{a}$ See Ref. 4.

$I_{\mathrm{cap}}=q V_{\mathrm{QW}} \omega \int_{E_{c}^{b}}^{\infty} \rho_{u}(E) f\left(E, E_{\mathrm{fn}}^{u}\right) \rho_{c}(E)\left(1-f\left(E, E_{\mathrm{fn}}^{c}\right)\right) d E$,

$I_{\mathrm{esc}}=q V_{\mathrm{QW}} \omega \int_{E_{c}^{b}}^{\infty} \rho_{c}(E) f\left(E, E_{\mathrm{fn}}^{c}\right) \rho_{u}(E)\left(1-f\left(E, E_{\mathrm{fn}}^{u}\right)\right) d E$,

where $q$ is the elementary charge, $V_{\mathrm{QW}}$ the $\mathrm{QW}$ volume, $\omega$ a constant related to the transition rate between a confined and an unconfined state (expressed in $\left.\mathrm{s}^{-1} \mathrm{eV} \mathrm{cm}^{3}\right), \rho_{c}\left(\rho_{u}\right)$ the confined (unconfined) state density, $f\left(E, E_{\mathrm{fn}}\right)$ the electron Fermi function, $E_{\mathrm{fn}}^{c}\left(E_{\mathrm{fn}}^{u}\right)$ the quasi-Fermi level for the confined (unconfined) carriers, and $E_{c}^{b}$ the energy of the edge of the barrier conduction band.

Expressions derived in Refs. 6 and 7 for modeling the capture and escape processes become equivalent to Eqs. (1) and (2) when one simply neglects the phonon energy and the dependence on the phonon number. Equations (1) and (2) can be solved analytically in the case of moderate injection levels in deep QWs, when the unconfined carrier quasi-Fermi level is far below the barrier band edge. For the conduction band, these solutions can be written as

$$
\begin{aligned}
& I_{\text {cap }}=q A \omega n_{L} \frac{m_{c}}{\pi \hbar^{2}} \frac{1}{4}\left(\frac{2 m_{u} k T}{\pi \hbar^{2}}\right)^{3 / 2} \exp \left[-\left(\frac{E_{c}^{b}-E_{\mathrm{fn}}^{u}}{k T}\right)\right], \\
& I_{\mathrm{esc}}=q A \omega n_{L} \frac{m_{c}}{\pi \hbar^{2}} \frac{1}{4}\left(\frac{2 m_{u} k T}{\pi \hbar^{2}}\right)^{3 / 2} \exp \left[-\left(\frac{E_{c}^{b}-E_{\mathrm{fn}}^{c}}{k T}\right)\right],
\end{aligned}
$$

where $m_{c}\left(m_{u}\right)$ is the effective mass of the confined (unconfined) carriers, $A$ the junction area, and $n_{L}$ the number of electron-confined levels.

In standard three-rate-equation QW laser models, ${ }^{3}$ the total capture time consists of the transport time along the confinement region and the intrinsic capture time, the latter scaled up by the volume factor $V_{\text {core }} / V_{\mathrm{QW}}$, with $V_{\text {core }}$ $\equiv A d_{\text {core }}$, and $d_{\text {core }}$ being the total thickness of the core region (confinement + barrier $+Q W$ ). In lasers with narrow confinement regions the capture process dominates over the transport, and therefore, the total capture time is directly proportional to the intrinsic capture time and to the volume factor. In this case, the dynamic capture and escape times are calculated from the first derivative of the net current flowing into the well $\left(I_{\text {net }} \equiv I_{\text {cap }}-I_{\text {esc }}\right)$ with respect to the unconfined $\left(n_{u}\right)$ and confined $\left(n_{c}\right)$ carrier densities, respectively, ${ }^{7}$ i.e., $q V_{\text {core }} / \tau_{\text {cap }} \equiv \partial I_{\text {net }} / \partial n_{u}$ and $q V_{\mathrm{QW}} / \tau_{\text {esc }} \equiv \partial I_{\text {net }} / \partial n_{c}$. Assuming for simplicity that there is only one confined level of electrons, the following expressions are obtained:

$$
\tau_{\text {cap }}=\frac{\pi \hbar^{2}}{\omega m_{c}} d_{\text {core }}
$$

$$
\tau_{\mathrm{esc}}=\frac{k T}{\omega} 4\left(\frac{\pi \hbar^{2}}{2 m_{u} k T}\right)^{3 / 2} \frac{\exp \left(\frac{q V_{b}}{k T}\right)}{1+\exp \left(\frac{E_{\mathrm{fn}}^{c}-E_{0}}{k T}\right)},
$$

where $q V_{b}$ is the energy difference between the first confined level and the edge of the barrier conduction band and $E_{0}$ is the energy of the confined level. If more than one confined energy level is considered, the analysis is similar except that expression (6) is replaced by a summation over all energy levels involved.

Equation (5) shows that $\tau_{\text {cap }}$ is constant with the injection level as a consequence of having considered $E_{\mathrm{fn}}^{u}$ to be far away from the barrier band edge. At low injection levels, the exponential term in the denominator of Eq. (6) is negligible with respect to unity, and therefore, $\tau_{\text {esc }}$ is also constant; the functional dependence on the barrier height is then identical to that given by the thermionic emission model. ${ }^{5} \mathrm{~A}$ decrease in the escape time with the injection level is predicted by Eq. (6) at high carrier densities due to the state filling effect, which enhances the reemission of carriers out of the QW. It is interesting to note that $\omega$ is a free parameter appearing in both $\tau_{\text {cap }}$ and $\tau_{\text {esc }}$ expressions, and therefore, the ratio $\tau_{\text {cap }} / \tau_{\text {esc }}$ can be calculated without needing additional parameters. It should also be noted that expressions (5) and (6) do not apply to very high injection conditions. However, in those cases, a relatively simple numerical integration of Eqs. (1) and (2) provides a good estimate of $\tau_{\text {cap }}$ and $\tau_{\text {esc }}$, compared to LO phonon interaction theory.

We have compared the predictions of the model with our previous experimental results on the escape time and on the ratio $\tau_{\text {cap }} / \tau_{\text {esc }}$ for InGaAs/GaAs QW lasers ${ }^{4,9,10}$ extracted from subthreshold high-frequency electrical impedance measurements. This technique provides either a value of the escape time or its lower/upper limit, depending on the ratio of $\tau_{\text {esc }}$ and the carrier lifetime. ${ }^{9}$ By analyzing samples with the same active material but different number of QWs, we were able to extract the ratio $\tau_{\text {cap }} / \tau_{\text {esc }}$. ${ }^{10}$ Table I summarizes the properties of active regions of the laser structures, together with the experimental values or estimated limits for $\tau_{\text {esc }}$ and for the ratio $\tau_{\text {cap }} / \tau_{\text {esc }}$. More details on the layer structure, growth conditions, and optical and electrical properties of the devices can be found in Refs. 4, 9, and 11 for samples A-C and in Refs. 4, 10, and 12 for samples D-F. 


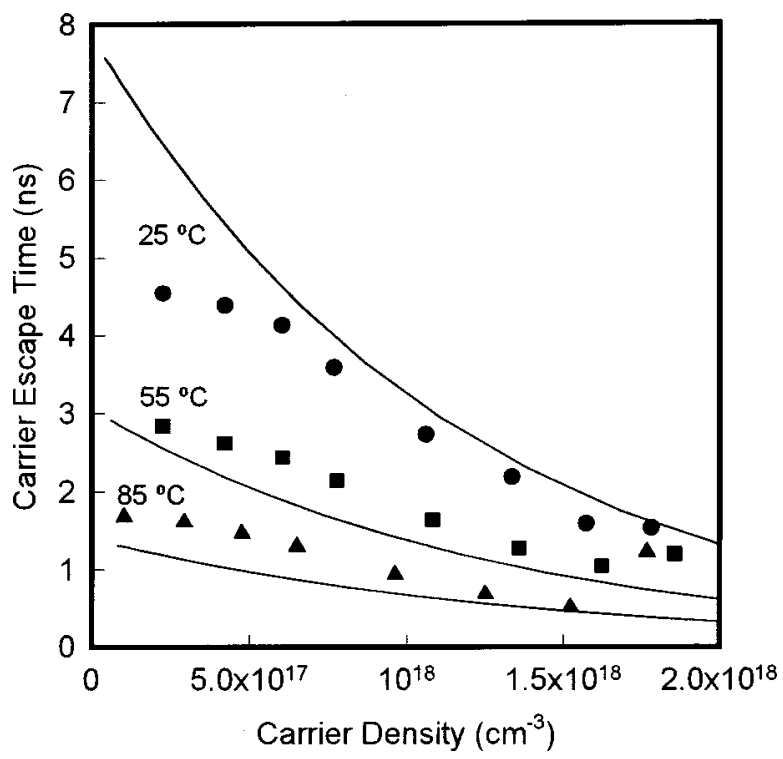

FIG. 2. Experimental (see Ref. 4) (points) and calculated (lines) carrier escape time vs carrier density for sample B at three different temperatures.

The measured values of $\tau_{\text {esc }}$ and of the ratio $\tau_{\text {cap }} / \tau_{\text {esc }}$ can be qualitatively understood considering that the electrons, not the holes, are responsible for the dynamic response of the devices. This follows from a comparison of $\tau_{\text {esc }}$ at a constant-carrier density for the $\operatorname{In}_{0.35} \mathrm{Ga}_{0.65}$ As lasers with different $p$-doping concentrations in the active regions (samples A-C). The doping produces a bandbending in the barrier regions which increases the electron effective barrier height, and hence, $\tau_{\text {esc }}$. Additionally, we observe that $\tau_{\text {esc }}$ is lower for samples with a lower In content due to the lower barrier height, and that the ratio $\tau_{\text {cap }} / \tau_{\text {esc }}$ decreases with the number of QWs (samples D-F) due to a decrease in the volume factor $V_{\text {core }} / V_{\mathrm{QW}}$. The experimental dependence of $\tau_{\text {esc }}$ on the injection current and the temperature for sample B is plotted in Fig. 2. As predicted by expression (6), $\tau_{\text {esc }}$ decreases with the injected carrier density due to the bandfilling effect. Since the quasi-Fermi level for electrons is much higher than that for holes due to their lower effective mass, this confirms again the predominant role of electrons in the dynamic response.

We have calculated $\tau_{\text {cap }}$ and $\tau_{\text {esc }}$ using expressions (5) and (6) and considering a single value for $\omega$ for all samples and temperatures. The calculations were performed for electrons using the standard values for their effective masses and the band offset, and assuming a single confined level. In the case of $p$-doped samples, the effective barrier height was calculated by self-consistently solving the Poisson and Schrödinger equations. The carrier-induced band-gap narrowing, which produces an increase in the barrier height, was also included in the calculations.

A value of $\omega=3.5 \times 10^{-7} \mathrm{eV} \mathrm{cm}^{3} \mathrm{~s}^{-1}$ yielded the best agreement with the complete set of experimental data. This value corresponds to a capture time of $0.5 \mathrm{ps}$ in the case of $\mathrm{In}_{0.35} \mathrm{Ga}_{0.65} \mathrm{As} \mathrm{QW}$ lasers, which is consistent with the maximum modulation bandwidth measured in similar devices [40 $\mathrm{GHz}$ (Ref. 13)]. Table I shows the calculated values of $\tau_{\text {esc }}$ and of the ratio $\tau_{\text {cap }} / \tau_{\text {esc }}$ at a confined carrier density of $10^{18} \mathrm{~cm}^{-3}$ for a set of samples. The agreement with the experimental data is good considering the simplicity of the model, the uncertainty in theoretical parameters, and the inaccuracy of experimental data. The calculated dependence of $\tau_{\text {esc }}$ on the carrier density and on the temperature is plotted in Fig. 2 in comparison with the experimental data. As it can be seen, the main fundamental trends are well reproduced by our simple model.

From the comparison between the experimental results with the predictions of the model, we conclude that the simple analytical expression (6) is enough to take into account the dependence of the escape time on the barrier height, the injection level, and the temperature. This implies that the main physical factor determining these dependencies is the number of carriers with energies higher than that of the barrier, and that the nature of the particular scattering mechanism which gives rise to the transition is not relevant.

In summary, we have presented a simple model that provides an analytical expression for the ratio of the carrier capture time to the escape time in QW lasers. The model predicts similar qualitative dependencies for the escape time as do more sophisticated models. Using a single fitting parameter, it yields a good agreement with a set of experimental results for InGaAs/GaAs QW lasers. We conclude that the main factor determining the escape time is the number of confined carriers with energies higher than that of the barrier. Furthermore, this model can be useful for analyzing, predicting, and simulating static as well as dynamic properties of QW lasers with different layer structures and working under different operating conditions, with the advantage of including the main physical effects involved in capture/escape processes without increasing the computing time.

This work has been supported by Comisión Interministerial de Ciencia y Technología (Spain), Project TIC-980418-C05. The work of one of the authors (M.C.) was supported by Ministerio de Educación y Ciencia, Spain, and by the Natural Sciences and Engineering Research Council (NSERC) of Canada.

${ }^{1}$ R. Nagarajan, M. Ishikawa, T. Fukushima, R. S. Geels, and J. E. Bowers, IEEE J. Quantum Electron. 28, 1990 (1992).

${ }^{2}$ S. C. Kan, D. Vassilovski, T. C. Wu, and K. Y. Lau, Appl. Phys. Lett. 62, 2307 (1993)

${ }^{3}$ L. A. Coldren and S. W. Corzine, Diode Laser and Photonic Integrated Circuits (Wiley, New York, 1995).

${ }^{4}$ I. Esquivias, B. Romero, S. Weisser, K. Czotscher, J. D. Ralston, E. C. Larkins, J. Arias, A. Schönfelder, M. Mikulla, J. Fleissner, and J. Rosenzweig, Proc. SPIE 2684, 17 (1996).

${ }^{5}$ H. Schneider and K. V. Klitzing, Phys. Rev. B 38, 6160 (1988)

${ }^{6}$ S. Kan, D. Vassilovski, T. C. Wu, and K. Lau, IEEE Photonics Technol. Lett. 4, 428 (1992).

${ }^{7}$ C. Y. Tsai, Y. H. Lo, R. M. Spencer, and L. F. Eastman, IEEE J. Sel. Top. Quantum Electron. 1, 316 (1995).

${ }^{8}$ M. Grupen and K. Hess, IEEE J. Quantum Electron. 34, 120 (1998).

${ }^{9}$ I. Esquivias, S. Weisser, B. Romero, J. D. Ralston, and J. Rosenzweig, IEEE J. Quantum Electron. 35, 635 (1999).

${ }^{10}$ B. Romero, I. Esquivias, S. Weisser, E. C. Larkins, and J. Rosenzweig, IEEE Photonics Technol. Lett. 11, 779 (1999).

${ }^{11}$ J. D. Ralston, S. Weisser, I. Esquivias, E. C. Larkins, J. Rosenzweig, P. J. Tasker, and J. Fleissner, IEEE J. Quantum Electron. 29, 1648 (1993).

${ }^{12}$ M. Mikulla, W. Benz, P. Chazan, J. Daleiden, J. Fleissner, G. Kaufel, E. C. Larkins, M. Maier, J. D. Ralston, J. Rosenzweig, and A. Wetzel, Inst. Phys. Conf. Ser. 145, 995 (1995).

${ }^{13}$ S. Weisser, E. C. Larkins, K. Czotscher, W. Benz, J. Daleiden, I. Esquivias, J. Fleissner, J. D. Ralston, B. Romero, R. E. Sah, A. Schönfelder, and J. Rosenzweig, IEEE Photonics Technol. Lett. 8, 608 (1996). 